\title{
Chest PHYSIOTHERAPY AFTER CARDIAC SURGERY - THE MISSING LINK
}

\author{
ABSTRACT: The physiotherapy literature pertaining to the treatment of cardiac surgery \\ patients in the period 1977 to 1995 was reviewed. The purpose of this review was to analyse \\ the results of the research and draw up guidelines for the physiotherapy treatment of cardiac \\ surgery patients. This review revealed that there is no indication for routine chest physio- \\ therapy in the uncomplicated cardiac surgery patient.
}

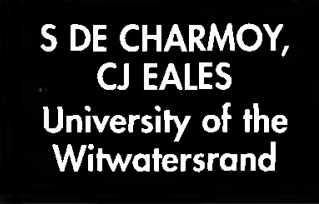

\section{KEYWORDS: CARDIAC SURGERY, CHEST PHYSIOTHERAPY, BREATHING EXERCISES}

\section{INTRODUCTION}

Open heart surgery and the use of cardiopulmonary bypass have long been recognised as creating a number of severe pulmonary abnormalities postoperatively. Until recently, post-operative chest physiotherapy was thought to play a role in reducing these abnormalities and returning pulmonary function to normal. The recent research in this area suggests that the routine use of postoperative chest physiotherapy in the uncomplicated patient is of no benefit.

This literature review was conducted to establish the trends in physiotherapy management of the post-operative cardiac patient over the last twenty years. Conclusions will be drawn from the literature and recommendations for practice made according to the findings.

\section{LITERATURE REVIEW \\ CHEST PHYSIOTHERAPY}

On return from theatre cardiac surgery patients typically spend the first 12 to 18 hours intubated and ventilated in the intensive care unit. Eales et al (1995) conducted a study to determine if routine physiotherapy for the intubated patient was indicated. They divided their patient population into three groups. The patients in all three groups were pre-oxygenated and suctioned. In addition the patients in Group Two were manually hyperinflated six times and then suctioned. The patients in Group Three received six manual hyperinflations together with chest wall vibrations, administered during the expiratory phase, plus suctioning. They found no significant differences in compliance, partial pressure of oxygen in arterial blood $\left(\mathrm{PaO}_{2}\right)$ and the partial pressure of oxygen in arterial blood to the fraction of inspired oxygen $\left(\mathrm{PaO}_{2} / \mathrm{FIO}_{2}\right.$ ratio), between any of the three groups after treatment. They concluded that a single physiotherapy treatment to the intubated post-operative cardiac patient was of no significant benefit.

In 1977 Varciu and Varciu studied a group of patients undergoing open heart surgery whom they divided into a high risk and low risk group. Patients considered at high risk for developing postoperative pulmonary complications had one or more of the following features:

- smokers or those who had

ceased to smoke in the previous

six weeks

- an FVC less than $80 \%$ and a

FEV $/$ FVC less than 75

- older than 60 years of age

Each of the above two groups was then further divided into an experimental and a control group. The experimental group was seen by a physiotherapist twice daily during which time the patients were treated with deep breathing exercises in various positions and were encouraged to cough. In addition they also received the routine ward regime. This included incentive spirometry two hourly, nebulisation four hourly and tuming, deep breathing and coughing every hour as administered by the nursing staff. The control group participated in the ward regime only.

Varciu and Varciu (1977) concluded that the use of breathing exercises in high risk patients reduces the incidence of post-operative pulmonary complications, but is of no benefit in the low risk group. The following factors should be borne in mind when interpreting this research.

It is not stipulated in the methodology whether the control groups received any input from the physiotherapist either preoperatively or post-operatively. In this study percutaneous catheters were used to assist in lung clearance in patients with excessive secretions who were unable to cough effectively. In the experimental high risk group, none of the patients required the use of a percutaneous catheter, in contrast 6 out of 13 patients in the control high risk group required their use. It is possible that the need to use percutaneous catheters arose as a result of the patients' lack of training or instruction in coughing, and not as a result of not having the breathing exercises. Lastly, the researchers omitted to mention the amount of active exercising in bed or walking, whether independently or assisted by the therapist, that the patients did post-operatively. The missing data from this research study may affect the interpretation of the results.

In a study by Iverson et al (1978) three groups of patients undergoing open heart surgery received one of three treatments. All three groups received instruction in breathing exercises and coughing to which was added either intermittent positive pressure breathing, blow bottles or incentive spirometry. Their results showed that the intermittent positive pressure breathing group fared the worst with a greater number of respiratory complications than the other two groups while the group using blow bottles had the fewest complications. The results also showed that none of the above techniques prevented atelectasis from occurring or improved it during the 72 hour study period.

Once again the interpretation of these results should be made with the following factors in mind. No mention is made of the patients' position during these treatments, or whether or not the patients were walking alone or with help at any stage. The definition of a pulmonary complication is not clearly stated and this makes comparisons with other studies difficult. The findings for the group who used incentive spirometry should be viewed with caution. The authors state that pump times for this group were significantly longer than those of the other two groups, a result of a change in operative procedure. Had the incentive spirometry group not had this confound- 
ing factor their results may have been different.

Gale and Saunders (1980) compared a Bartlett-Edwards incentive spirometer to intermittent positive pressure breathing in a group of patients who had undergone open heart surgery. A regime of pre-operative training with the chosen modality was followed with four hourly post-operative use of the modality for three days. They concluded that incentive spirometry is not significantly better than intermittent positive pressure breathing in preventing post-operative atelectasis. Following_both_forms of treatment there, was a trend to hypoxaemia which was slightly greater in the intermittent positive pressure breathing group.

The results of this study are difficult to compare with other studies as no details are given concerning chest physiotherapy. It is thus presumed that patients received no chest physiotherapy. There is also no mention of walking or active bed exercise programmes that patients may have followed during this time. It seems unlikely that a regime of ten incentive spirometry breaths, or twenty intermittent positive pressure breaths alone, in a twenty minute treatment session is insufficient to have an effect on atelectasis.

Oulton et al (1981) considered whether different incentive breathing devices added any benefit to a regime of standard chest physiotherapy, in a group of coronary artery surgery patients. They compared chest physiotherapy alone (which consisted of encouragement to cough, deep breathing, postural drainage, vibration and percussion) to chest physiotherapy plus either a Triflo spirometer or a Spirocare spirometer. All patients were taught how to use their chosen device pre-operatively. Their results showed that the group using_the $\sqrt{\text { Spirocare }}$ spirometer had less post-operative atelectasis on chest $x$-rays throughout the first four post-operative days than the other two groups. This was thought to be due to the Spirocare spirometer having an additional visual stimulus to hold maximum inspiration for three seconds. With the Triflo spirometer a fast flow rate can bring about a relatively large volume change, and inspiratory hold is not encouraged.

It should be noted that postural drainage positions and the position of the patient while using the spirometers were not described. In addition, no information is given about patient mobility. The authors state that after five patients had been entered into each group it was obvious that the group using the Spirocare spirometer was faring the best. In interpreting the results of this research, it is important to note that the groups were not well matched for age and this may have influenced the results. The mean age of the chest physiotherapy group was 45 years while the group using the Spirocare had a mean age of 60 years.

Stock et al (1984) compared continuous positive airway pressure, incentive spirometry and conservative chest physiotherapy in a group of elective open heart surgery patients $(n=38)$. Conservative therapy was considered four to five maximal inhalations, huffing and instruction to "cough heartily". No details were given about patient positioning for treatment or how soon the patients were made to walk. Each treatment lasted fifteen minutes and occurred every two waking hours for the first three post-operative days. This could lead to confounding results as it is felt that in clinical practice the effect of a treatment should be evaluated on the clinical outcomes and not be determined by a time period. They concluded that neither conservative chest physiotherapy, incentive spirometry or continuous positive airway pressure improved the restrictive lung function defect within the first 72 hours post-operatively.

Oikkonen et al (1991) found similar results in a study in which intermittent positive pressure breathing or incentive spirometry were given together with conventional chest physiotherapy in a group of patients who had undergone coronary artery surgery $(n=52)$. This conventional chest physiotherapy consisted of "breathing techniques, deep diaphragmatic ventilation and efficient coughing". The patients were trained in these techniques for two days pre-operatively. Post-operatively the patients received this conventional physiotherapy a minimum of once a day. They also received intermittent positive pressure breathing on four occasions during the day or, incentive spirometry every alternate waking hour. They concluded that the incidence of atelectasis in both groups increased during the study period. In other words, intermittent positive pressure breathing, incentive spirometry and conventional chest physliotherapy were unable to prevent or improve the post-operative atelectasis that occurs following open heart surgery.
Once again this research does not mention a bed exercise programme or at what stage the patients walked. The position in which the physiotherapy was done in, is also not included.

It is difficult to analyse the data because of the inconsistency of the studies. Some of these difficulties have been highlighted already, those not mentioned before will now be discussed. The patient groups for the different studies were not standardised. Some groups consisted of patients undergoing different kinds of surgical procedures, while others were patients all undergoing the same procedure. This may have resulted in different results as the problems of a valvular surgery patient are different from those of a coronary artery surgery patient. The inclusion and exclusion criteria are not always clearly stated and differ between studies. As has been mentioned previously, walking the patient has not been addressed, and thus is a poorly controlled variable. Patient position for "physiotherapy" techniques are also not consistently recorded and thus could play a role in the results of these studies. Chest physiotherapy it would seem has multiple definitions as no two studies used the same chest physiotherapy regime. Control groups receiving no physiotherapy were never considered, and thus it is difficult to isolate the effect of physiotherapy.

\section{THE ROLE OF ACTIVE EXERCISE AND WALKING THE PATIENT}

Dull and Dull (1983) compared early mobilisation alone to early mobilisation plus breathing exercises or incentive spirometry. Early mobilisation was defined as "ankle circumduction, range of motion to all extremities, three maximal coughs, and encouragement and assistance to turn from side to side, sit up, or stand up". The study group included 29 patients who had coronary artery surgery and 20 who had valve replacement surgery. They found that neither of the "added" modalities (incentive spirometry or breathing exercises) were beneficial to the early mobilisation programme alone. In addition, none of the three programmes improved the lung function changes seen post-operatively.

For the purposes of this study a pulmonary complication was defined as:

- a temperature elevation of $4^{\circ} \mathrm{F}$ above the mean pre-operative 
temperature

- a temperature elevation of 2 to $3^{\circ} \mathrm{F}$ above the mean pre-operative temperature in addition to abnormal auscultatory findings

- purulent sputum.

Using these definitions the authors found that $77 \%$ of the patients who underwent coronary artery surgery, and $92 \%$ of the valvular surgery patients developed a post-operative complication during their respective treatment programmes. They thus concluded that none of the treatment programmes was effective at preventing post-operative pulmonary complications. It is also possible that the definition of a post-operative pulmonary complication was too broad thus accounting for between $77 \%$ and $92 \%$ of patients developing such a complication.

In a study of 110 white males undergoing coronary artery surgery three different treatment protocols were assessed (Jenkins et al 1989, 1990). The study population was divided into three groups. All the study participants were seen preoperatively by a physiotherapist and were taught huffing, coughing with sternal support and active upper and lower limb exercises. The need to move about post-operatively and expectorate bronchial secretions was also explained. This was the only physiotherapy that the patients in the control group received post-operatively. The patients in the other two groups received either localised breathing exercises, (with vibrations and percussion in a postural drainage position if deemed necessary) or incentive spirometry. The patients in both these groups were taught their respective techniques pre-operatively and encouraged to practise them. They found that adding breathing exercises or incentive spirometery to the programme of the control group, did not alter their treatment outcome. The authors recommended that uncomplicated coronary artery surgery patient be taught and helped with a mobility regime.

A further study by Jenkins et al (1994), in which patients undergoing coronary artery surgery, were simply encouraged to take deep breaths, cough and were mobilised by the nursing and surgical staff revealed results similar to the studies cited above. The incidence of respiratory complications post-operatively remained low $(9 \%)$ despite the lack of chest physiotherapy. Patients excluded from this study included those who had had previous coronary artery surgery or pulmonary surgery, and those who had a pre-operative respiratory abnormality.

Stiller et al. (1994) included a control group in their study which received no pre-or post-operative physiotherapy. This was the first study in which physiotherapy was completely excluded. The control group followed the normal mobilisation protocol of the hospital which included sitting out of bed on day two and walking from day three. The study population was made up exclusively of patients undergoing coronary artery surgery. The results from this study were in agreement with the above two studies. The incidence and severity of hypoxaemia, fever, chest $\mathrm{X}$ ray abnormalities and significant pulmonary complications were not notably higher for the control group. Patients excluded from this study included those who were mechanically ventilated for more than 24 hours post-operatively, and those who developed a neurological or cardiac complication that rendered them unable to participate in the study. The recommendation of this study is that all patients be continually assessed for clinically significant pulmonary complicafions, and treated with physintherapy if and when the need arises. Routine postoperative chest physiotherapy is not indicated in this patient population.

Stiller et al (1994) make the following point. Although the control group received no pre-operative physiotherapy they did watch a video pre-operatively which mentions chest physiotherapy. In the process of giving informed consent to participate in the study, the patients were made aware of the rationale for doing breathing exercises and coughing postoperatively. Both these factors may have affected the behaviour in the control group.

In 1995 Stiller et al investigated whether the incidence of clinically significant pulmonary complications had increased since the recommendation that routine post-operative physiotherapy was not necessary in the uncomplicated coronary artery surgery patient. The 1995 study included all patients undergoing heart surgery requiring cardiopulmonary by-pass. The only difference in this study from the 1994 study was that it included 13 patients who had undergone valve surgery without coronary artery surgery. Clinically significant pulmonary complications were found in $7.1 \%$ (nine out of 127 patients) of the total patient population. An important consideration in this study is that all patients undergoing cardiac surgery were included and thus patients with significant pre-operative risk factors were also included.

\section{CONCIUSIONS}

It would seem then that the following conclusions can be drawn from the literature described previously.

TABLE I

\begin{tabular}{|c|c|c|c|c|}
\hline & Jenkins (1989) & Jenkins (1994) & Stiller (1994) & Stiller (1995) \\
\hline Sex: $M / F(n)$ & $110 / 0$ & $165 / 0$ & $98 / 29$ & $98 / 22$ \\
\hline Age (years)' & $55.7 \pm 8.0$ & $58.7 \pm 9.6$ & $61.3 \pm 9.9$ & $62.0 \pm 9.4$ \\
\hline CABG & 110 & 165 & 115 & 115 \\
\hline Valve Replacement & 0 & 0 & 12 & 5 \\
\hline $\begin{array}{l}\text { No. of patients } \\
\text { with Pulmonary } \\
\text { complications }\end{array}$ & $11(10 \%)$ & $5(3.4 \%)$ & $9(7.1 \%)$ & $9(7.5 \%)$ \\
\hline
\end{tabular}


A considerable amount of research has been conducted into the effects of various post-operative physiotherapy treatment protocols for the cardiac surgery patient.

The trend in the recent research is away from routine physiotherapy treatments with the emphasis on assessment and appropriate intervention. This is possibly due to the improvement in anaesthetic techniques, cardiopulmonary bypass equipment and the use there of, improved surgical techniques and shorter periods of mechanical ventilation post-operatively.

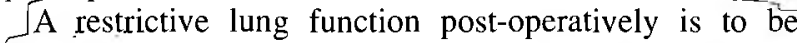
expected and physiotherapy seems to have little impact on this. enkins et al (1990) found that the patients who had walked prior to their lung function test on the second postoperative day had a significant increase in functional residual capacity as compared to those patients who had not walked. This_would support the rationale behind a programme of early mobilisation and walking.

No one treatment technique or device has been shown to be superior to another. This is due to the inability to compare different studies as a result of inconsistencies in study protocols.

Based on the results of the Stiller et al (1995) study preoperative physiotherapy is also of no benefit in preventing clinically significant pulmonary complications post-operatively.

Physiotherapy is not indicated in a group of patients undergoing elective cardiac surgery who are walking_well from day two onwards. This conclusion does not include patients who have a complicated post-operative course. This would include patients who are intubated for longer than 24 hours post-operatively and patients who develop neurological complications.

Patients should be assessed daily in the post-operative period for clinically significant pulmonary complications.

Signs and symptoms of a significant pulmonary complication should include:

- a temperature of greater than $38.50 \mathrm{C}$

- the necessity for antibiotics post-operatively over and above the usual treatment.

- radiological evidence of significant collapse or consolidation signs of respiratory distress.

In summary the following "missing links" have been identified.

There is currently no evidence to support routine chest physiotherapy after cardiac surgery.

There is no role for preventative chest physiotherapy in the post-operative cardiac surgery patient.

\section{REFERENCES}

1. Dull JL and Dull WL. Are maximal inspiratory breathing exercises or incentive spirometry better than early mobilization after cardiopulmonary bypass. Physical Therapy 1983, 63: 655 - 659 .

2. Eales CJ, Barker M and Cubberley NJ. Evaluation of a single chest physiotherapy treatment to post-operative, mechanically ventilated cardiac surgery patients. Physiotherapy Theory and Practice 1995, 11: 23 - 28

3. Gale GD and Sanders DE. Incentive spirometry: Its value after cardiac surgery. Canadian Anaesthetists' Society Journal 1980, 27: 475 - 480 .

4. Iverson LIG, Ecker RR, Fox HE and May IAA comparative study of IPPB, the incentive spirometer, and blow bottles: The prevention of atelectasis following cardiac surgery. The
Annals of Thoracic Surgery 1978, 25: 197-200

5. Jenkins SC, Soutar SA, Loukota JM, Johnson LC and Moxham J. Physiotherapy after coronary artery surgery: Are breathing exercises necessary? Thorax 1989, 44: 634-639

6. Jenkins SC, Soutar SA, Loukota JM, Johnson LC and Moxham J. A comparison of breathing exercises, incentive spirometry and mobilisation after coronary artery surgery. Physiotherapy Theory and Practice 1990, 6: 117-126

7. Jenkins S, Akinkugbe Y, Corry G and Johnson L. Physiotherapy management following coronary artery surgery. Physiotherapy Theory and Practice 1994, 10: 3-8

8. Oikkonen M, Karjalainen K, Kädärä V, Kuosa R and Schavikin L. Comparison of incentive spirometry and intermittent positive pressure breathing after coronary artery bypass graft. Chest 1991, 99: 60 - 65 9. Oulton J L, Hobbs GM and Hicken P. Incentive breathing devices and chest physiotherapy: A controlled trial. The Canadian Journal of Surgery 1981, 24: 638-640

10. Stiller K, Montarello J, Wallace M, Daff M, Grant R, Jenkins S, Hall $B$ and Yates $H$. Are breathing and coughing exercises necessary after coronary artery surgery? Physiotherapy Theory and Practice 1994, 10: 143-152

11. Stock MC, Downs JB, Cooper RB, Lebenson IM, Cleveland J, Weaver DE, Alster JM and Imrey PB. Comparison of continuous positive airway pressure, incentive spirometry, and conservative therapy after cardiac operations. Critical Care Medicine 1984, 12: $969-972$

12. Vraciu JK and Vraciu RA. Effectiveness of breathing exercises in preventing pulmonary complications following open heart surgery. Physical Therapy 1977, 57: 1367-1371

L
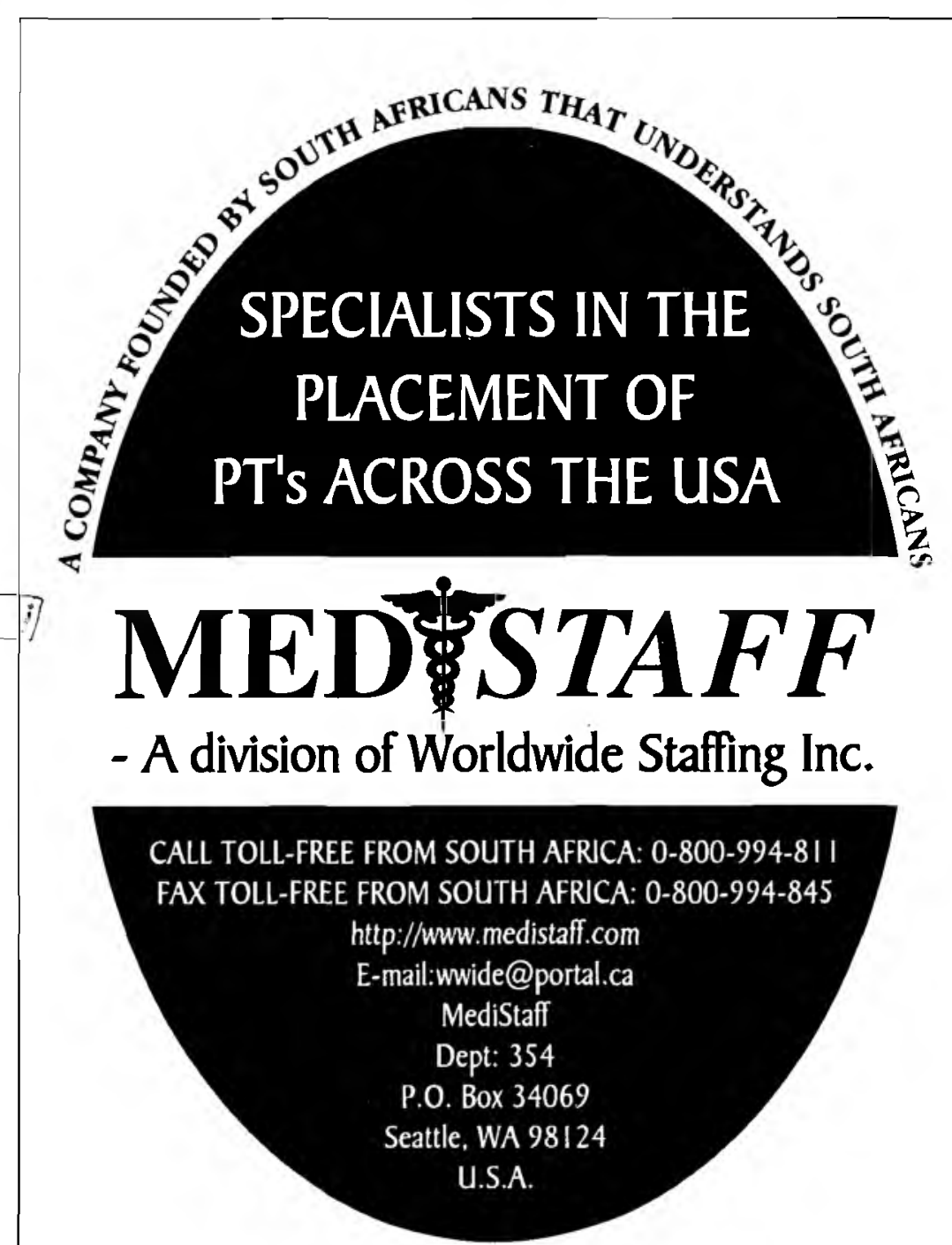